\title{
Uso de tecnologias na capacitação de agentes comunitários de saúde: enfrentamento da COVID-19
}

\author{
Use of technologies in the training of community health agents: coping with COVID-19 \\ Uso de tecnologías en la formación de agentes de salud comunitarios: afrontando COVID-19
}

Recebido: 13/01/2022 | Revisado: 21/01/2022 | Aceito: 25/01/2022 | Publicado: 26/01/2022

ORCID: https://orcid.org/0000-0002-4644-274X Universidade Federal de São João del-Rei, Brasil

E-mail: danielcortez@ufsj.edu.br

Elen Soraia de Menezes Cabral

ORCID: https://orcid.org/0000-0002-3147-5151 Universidade Federal de São João del-Rei, Brasil

E-mail: elenmenezes@ufsj.edu.br

Raruza Keara Teixeira Gonçalves

ORCID: https://orcid.org/0000-0002-2416-7628

Universidade Federal de São João del-Rei, Brasil

E-mail: raruzakeara@ufsj.edu.br

Andreza Oliveira-Cortez

ORCID: https://orcid.org/0000-0002-7000-0490

Universidade Federal de São João del-Rei, Brasil

E-mail: andrezaohcortez@gmail.com

Fabricio Rodrigues dos Santos

ORCID: https://orcid.org/0000-0001-5100-1036

Universidade Federal de São João del-Rei, Brasil

E-mail: fabriciorosan@hotmail.com

Dárlinton Barbosa Feres Carvalho

ORCID: https://orcid.org/0000-0003-3844-0178

Universidade Federal de São João del-Rei, Brasil

E-mail: darlinton@ufsj.edu.br

Eliete Albano de Azevedo Guimarães

ORCID: https://orcid.org/0000-0001-9236-8643

Universidade Federal de São João del-Rei, Brasil

E-mail: elietealbano@ufsj.edu.br

Valéria Conceição de Oliveira

ORCID: https://orcid.org/0000-0003-2606-9754

Universidade Federal de São João del-Rei, Brasil

E-mail: valeriaoliveira@ufsj.edu.br

\begin{abstract}
Resumo
A pandemia da COVID-19 exigiu da Atenção Primária à Saúde uma readequação no cuidado no que tange a abordagem do Agente Comunitário à Saúde quanto às visitas domiciliares e demais atividades. Este artigo teve o objetivo de relatar a experiência da criação e desenvolvimento de vídeos educativos, numa parceria entre a Universidade e a Atenção Primária à Saúde, adotados como instrumentos facilitadores para o trabalho dos Agentes Comunitários de Saúde, no enfrentamento à pandemia da COVID-19. A metodologia aplicada refere-se a um relato de experiência de inovação tecnológica da elaboração de vídeos educativos por professores universitários e discentes, destinados ao Agentes Comunitários de Saúde em sua nova rotina de trabalho. Consideraram-se as medidas, para contenção da epidemia, oriundas de órgãos governamentais e sanitários, abrangendo demandas institucionais, profissionais e comunitárias. Os vídeos foram disponibilizados para a coordenação da APS de cada município acompanhado de um documento informativo sobre os conteúdos abordados em cada vídeo.Para a divulgação, levou-se em conta a importância de dar visibilidade às informações de interesse público definidas por diretrizes do sistema de saúde durante o período de isolamento social. O trabalho possibilitou a realização de parcerias com secretarias municipais de saúde, as quais instrumentalizaram seus Agentes Comunitários de Saúde com os produtos deste trabalho. Os canais institucionais de comunicação da Universidade Federal de São João del-Rei, bem como as plataformas de mídias sociais dos envolvidos e apoiadores, também se tornaram espaço para divulgação.

Palavras-chave: Agentes comunitários de saúde; Atenção primária à saúde; COVID-19; Filme e vídeo educativo; Infecções por Coronavírus.
\end{abstract}




\begin{abstract}
The COVID-19 pandemic required from Primary Health Care a readjustment in care regarding the approach of the Community Health Agent regarding home visits and other activities. This article aimed to report the experience of creating and developing educational videos, in a partnership between the University and Primary Health Care, adopted as facilitating instruments for the work of Community Health Agents in the face of the COVID-19 pandemic. . The applied methodology refers to an experience report of technological innovation of the elaboration of educational videos by university professors and students, destined to the Community Health Agents in their new work routine. Measures to contain the epidemic were taken from government and health agencies, covering institutional, professional and community demands. The videos were made available for the coordination of the APS of each municipality, accompanied by an informative document on the contents covered in each video. health during the period of social isolation. The work made it possible to form partnerships with municipal health departments, which provided their Community Health Agents with the products of this work. The institutional communication channels of the Federal University of São João del-Rei, as well as the social media platforms of those involved and supporters, also became a space for dissemination.
\end{abstract}

Keywords: Community health workers; Primary health care; COVID-19; Instructional film and video; Coronavirus infections.

\title{
Resumen
}

La pandemia de COVID-19 requirió de la Atención Primaria de Salud un reajuste en la atención en cuanto al abordaje del Agente Comunitario de Salud en cuanto a visitas domiciliarias y otras actividades. Este artículo tuvo como objetivo relatar la experiencia de creación y desarrollo de videos educativos, en alianza entre la Universidad y la Atención Primaria de Salud, adoptados como instrumentos facilitadores para el trabajo de los Agentes Comunitarios de Salud, frente a la pandemia de la COVID-19. La metodología aplicada se refiere a un relato de experiencia de innovación tecnológica en la elaboración de videos educativos por parte de docentes y estudiantes universitarios, destinados a los Agentes Comunitarios de Salud en su nueva rutina laboral. Se tomaron medidas de contención de la epidemia desde los organismos gubernamentales y de salud, cubriendo las demandas institucionales, profesionales y comunitarias. Los videos fueron puestos a disposición de la coordinación de las APS de cada municipio, acompañados de un documento informativo sobre los contenidos que abarca cada video salud durante el período de aislamiento social. El trabajo posibilitó la formación de alianzas con las secretarías municipales de salud, que pusieron a disposición de sus Agentes Comunitarios de Salud los productos de este trabajo. Los canales de comunicación institucionales de la Universidad Federal de São João del-Rei, así como las plataformas de redes sociales de los involucrados y simpatizantes, también se convirtieron en un espacio de difusión.

Palabras clave: Agentes comunitarios de salud; Atención primaria de salud; COVID-19; Película y video educativos; Infecciones por Coronavirus.

\section{Introdução}

A pandemia da COVID-19, gerou a necessidade de estabelecer normas emergenciais de convívio social como distanciamento e isolamento, consideradas medidas preventivas eficazes, para a diminuição da transmissão do vírus e, consequente, contenção das ondas epidêmicas (Aquino et al., 2020; Bavel et al. 2020).

O trabalho da vigilância e assistência à saúde na Atenção Primária à Saúde, assim como em outros níveis, associados ao fomento à pesquisa, desenvolvimento e inovação, têm direcionado ações que fortalecem o Sistema Único de Saúde (SUS) com o intuito de reduzir a transmissão do vírus, assim como complicações pela doença (Brasil, 2020).

Neste interim, destaca-se o papel do Agente Comunitário de Saúde (ACS), profissional da equipe da Estratégia Saúde da Família (ESF) que se relaciona mais estreitamente com a população de sua área de abrangência, destacando-se como agenciador de atividades emancipadoras e comunicativas, fundamentadas nos preceitos da Educação Popular em Saúde (EPS) (Maciel et al., 2020).

Espera-se desse profissional, competência cultural e orientação comunitária, entre equipe e comunidade. Isso se materializa no relacionamento cotidiano dos ACS com as pessoas de sua área de abrangência, por meio da visita domiciliar, importante ferramenta para informar, obter informações e gerar comunicação (Faria \& Paiva, 2020). A realização da visita domiciliar expõe o ACS, risco de adoecimento pelo Coronavírus e, nesse sentido, o Ministério da Saúde preconizou “Recomendações para adequação das ações frente à atual situação epidemiológica de COVID-19 (Brasil, 2020)". Tal 
documento ressalta a importância de considerar alguns cuidados que garantam a segurança da população e do profissional.

Para minimizar os riscos e a vulnerabilidade dos ACS, manter o vínculo com a comunidade sem prejuízo das atividades, faz-se necessário a busca de tecnologias inovadoras que apoiem e facilitem os ACS junto às suas ações com a comunidade (Maciel et al., 2020; Maciazeki-Gomes el al., 2016).

$\mathrm{O}$ avanço tecnológico propiciou uma mudança nos aspectos culturais de relacionamento entre as pessoas, em que os indivíduos no ambiente virtual agem de forma ativa e participativa, bem como a ampla adesão da população às novas tecnologias.

As novas práticas tornaram-se imprescindíveis para viabilizar as atividades do quotidiano. A sociedade precisou adaptar-se ao modo de vida online (conectado à rede) nas mais diversas situações, como a: busca por informação, trabalho em casa, reuniões, estudos, compras, relacionamentos familiares e afetivos, lazer, saúde e entretenimento (Gonçalves Caldeira Brant Losekann \& Cardoso Mourão, 2020).

O enfrentamento a pandemia da COVID-19 chama a atenção para o uso consciente e mobilizador de novas práticas comunicativas para as ações de caráter sanitário e educacional. As tecnologias do virtual assumem papel preponderante por meio de seus recursos hipermidiáticos, instrumentos de verdadeira importância para processos de aprendizagem que concedem aos usurários a competência de construir e reconstruir instâncias do conhecimento e da informação (Junior \& Monteiro, 2020).

A promoção da comunicação por meio de vídeos educacionais voltados para o trabalho do ACS com a comunidade, especialmente no enfrentamento à epidemia da COVID-19, é uma estratégia em potencial para apoiar as atividades de vigilância em saúde. A construção de vídeo educativo apresenta-se como instrumento didático e tecnológico que proporciona conhecimento e torna a interação educador-aprendiz efetiva (Dalmolin et al., 2016). O vídeo é uma tecnologia tem o potencial de fazer parte do cotidiano, ser atratividade e contextualizado, motivando a aprendizagem (Costa \& Mattos, 2016).

Este artigo buscou relatar a experiência vivenciada por docentes e discentes da Universidade Federal de São João Del Rei - UFSJ na criação, desenvolvimento e divulgação de vídeos educacionais de modo que pudessem ser adotados como instrumentos facilitadores do trabalho do ACS com a comunidade, no enfrentamento à epidemia da Covid-19.

A relevância deste artigo está em relatar uma experiência, envolvendo docentes e discentes das áreas de saúde, comunicação e jornalismo e computação numa proposta do uso de Novas Tecnologias da Informação e Comunicação (NTIC) para a criação de ferramentas digitais que têm a dupla potencialidade: capacitar e instrumentalizar o ACS para o seu trabalho, considerando as restrições de contato social durante a pandemia.

As parcerias institucionais entre ensino e serviço são essenciais para a produção de tecnologias inovadoras para implementar as práticas de saúde e capacitar o profissional. A crise causada pela pandemia na sociedade, deflagra a necessidade de ações coletivas, transversais e interdisciplinares para a prevenção e o combate à COVID-19.

\section{Metodologia}

Trata-se de relato de experiência de inovação tecnológica realizada em uma parceria entre as Secretarias de Saúde Municipais da Macrorregião Oeste de Minas Gerais e a UFSJ Campus Centro Oeste (CCO) no período de abril de 2020 a junho de 2021. Destaca-se que com a implantação dos Cursos de Enfermagem e Medicina no CCO em 2008, teve início o trabalho integrado da universidade com municípios desta Macrorregião. Como destaque da integração, a unidade curricular Prática de Integração Ensino Serviço e Comunidade- (PIESC) prevê a inserção do aluno desde o primeiro período nos serviços de saúde, respeitando as limitações do aluno e as questões éticas de sua atuação junto aos usuários e pacientes. É uma das propostas sociais da Universidade estabelecer parcerias institucionais e realizar projetos em colaboração que repercutam na melhoria dos serviços de saúde e colaborem para a formação acadêmica dos discentes. 
Em virtude da pandemia COVID-19 e da parceria já instituída entre a UFSJ e a Atenção Primária à Saúde- (APS) dos municípios da Macrorregião Oeste de Minas Gerais, surgiu uma demanda da coordenadora da APS da Superintendência Regional de Saúde, frente à necessidade de instrumentalização e apoio ao trabalho dos ACS, no período da epidemia. Para tanto, a UFSJ propôs para a capacitação, a adoção de estratégias inovadoras, não convencionais, pautadas em modelos de ensino-aprendizagem integradores e participativos, desvinculando-se do modelo de ensino ou capacitações tradicionais (Rodrigues et al., 2021).

As perspectivas inovadoras pautam-se no ideal construtivista, ou seja, o conhecimento não se configura em uma cópia da realidade, mas em uma construção do ser humano. Nele, o processo ensino-aprendizagem é compreendido como um todo e considera-se que o ouvinte seja um sujeito ativo e protagonista junto ao educador que tem o papel de facilitador (Rodrigues et al., 2021).

Diante disso, uma equipe multidisciplinar composta por seis professores das áreas de saúde, tecnologia (ciências da computação) e comunicação e um grupo de estudantes da graduação e pós-graduação da UFSJ, organizaram uma proposta com o intuito de capacitar o ACS para o enfrentamento da pandemia COVID-19, facilitando e otimizando o trabalho do mesmo, durante a quarentena. Simultânea e consequentemente, o trabalho pretendia empoderar a população da área de abrangência quanto às medidas de prevenção e controle da doença ao acessar o mesmo produto utilizado pelos ACS. O trabalho interdisciplinar envolve originalidade, criatividade e cooperação para o enfrentamento de situações reais como a pandemia do COVID-19.

Durante o desenvolvimento do trabalho, além da entrega dos vídeos diretamente aos ACS e gestores municipais, a equipe percebeu que o projeto poderia atingir uma abrangência maior se os vídeos criados fossem compartilhados em plataformas de mídias sociais como WhatsApp e Instagram.

Nesse contexto, uma questão que é digna de destaque é a mobilidade proporcionada pelos celulares. Os suportes portáteis tornaram-se verdadeiras extensões dos nossos corpos físicos, incidindo de forma imediata no modo de vida da sociedade da informação (Carraro et al., 2020). Para a maioria dos seus usuários, esse aparelho, pequeno e leve, conecta-os às pessoas em qualquer lugar, a qualquer momento, ou seja, ao mundo. A assincronia nas comunicações proporcionada pelo ambiente digital, permite que o usuário decida em qual momento acessará determinado conteúdo. Fato, que promove a liberdade de escolha na interação. Somado a isso, a atualização constante das informações nas formas mais diversas (texto, imagem, som, vídeo) oferta um rico repertório para o deleite do usuário (Carraro et al., 2020; Junior \& Monteiro, 2020).

O trabalho relatado utilizou recursos presentes nesses novos suportes e mídias para criar produções audiovisuais e narrativas educativas de caráter sanitário de qualidade. Dessa forma, os vídeos produzidos apresentam elementos audiovisuais destinados à mídia móvel.

\section{Resultados e Discussão}

O vídeo faz parte de uma prática, que se tornou dominante culturalmente a partir dos anos 1980, colaborando com produções autorais, com registros domésticos (gravações de aniversários e formatura, por exemplo), com a autonomia criativa de profissionais e videomakers (produtores independentes que trabalhavam no mercado de vídeo, criando trabalhos autorais e comerciais. Foi um movimento vertiginoso na década de 1980 quando jovens recém-formados nos cursos de comunicação social puderem explorar as novas tecnologias do circuito eletrônico) e de indivíduos comuns. O vídeo inaugura uma era em que câmeras mais leves e suportes portáteis, como videotape e fita cassete, redefiniram um novo caminho para o audiovisual (Machado, 2007).

É importante destacar, que o vídeo alterou a relação com o tempo devido à emissão e recepção da mensagem serem 
instantâneas, exemplo disso são as transmissões ao vivo, a liberdade de improviso, a valorização do aqui e agora e a capacidade de transformar, por meio da junção de imagens e de som, um fragmento da realidade em um acontecimento compartilhado socialmente (Jesus Gonçalves et al., 2020).

Além disso, o vídeo na era digital confirma sua natureza híbrida e experimental. O vídeo pode ser considerado revigorante e sua interação com o público e com que o produz, permite que as diversas oportunidades de sua experimentação promovam espaço para novos lugares e linguagens, redefinidos pelas redes e internet (Dutra Pillar \& Campos, 2021).

Aplicativos dotados de inteligência artificial colaboram com a mixagem de imagens, criação de arte e texto, legendas, plano, formato e ângulo, permitindo edições com apenas alguns clicks nos painéis de comando dos programas. Um repertório enorme para eleger elementos estéticos como cor, formato de letras, movimento das imagens e recortes e composições entre áudio e texto são ofertados nessas plataformas hipermidiáticas (Junior \& Monteiro, 2020).

O desafio do trabalho foi unir essa gama de possibilidades tecnológicas com o propósito: de apoiar e dar suporte ao trabalho do ACS. Neste sentido, buscou-se expor o conteúdo para comunicação por meio de vídeos de forma dinâmica, leve, empática e técnica: linguagem audiovisual estruturada em quatro partes fundamentais: pesquisa, roteiro, produção e edição.

Na pesquisa, a construção dos vídeos educativos foram baseados no material instrutivo do Ministério da Saúde - MS, intitulado "Recomendações para a adequação das ações dos Agentes Comunitários de Saúde frente à atual situação epidemiológica referente ao COVID-19" (Brasil, 2020).

$\mathrm{Na}$ roteirização, definimos os recursos estéticos (criação de artes comuns às temáticas dos vídeos, formatos para veiculação dos vídeos, resolução de imagem), definição das imagens adotadas que compatibilizavam com o áudio (gravado pelos próprios alunos-produtores em aplicativos dos celulares pessoais), escolha dos aplicativos e programas de edição e, por fim, a gravação dos próprios alunos, que deveriam realizar uma passagem no início de cada vídeo, afim de gerar identificação com o receptor dos vídeos.

Os dez vídeos criados para a divulgação do projeto respeitaram os mesmos padrões técnicos, tais como: O formato de vídeo para Android/ IOS H.264 e de áudio MP4 stereo e a resolução 16:9, que diz respeito à proporção de pixels horizontais e verticais durante a exibição de tela e é a resolução convencionalmente usada pela plataforma Youtube.

A padronização dos vídeos obedeceu também a outros critérios, como categorização temática, a produção de vídeos curtos com até três minutos de duração, adoção de uma mesma identidade visual, abertura e encerramento com a mesma assinatura, ou seja, com as mesmas artes e logomarcas das instituições promotoras do material audiovisual.

Outra questão que merece destaque é a junção entre os recursos estéticos e as finalidades interativas presentes nos vídeos. As categorias temáticas diferenciavam-se através das cores nas aberturas e encerramentos dos vídeos com o intuito de sinalizar para o público-alvo a mudança temática, priorizando o caráter informativo e pedagógico dos mesmos. E, finalmente, para promover a interação fácil com o material educativo dos vídeos, recorreu-se ao uso da $\neg \neg$ hashtag\#zerocovid19 como título padrão dos vídeos veiculados, aproximando-os da linguagem comumente usada no ambiente das redes sociais. A hashtag é um termo associado a assuntos, histórias, ideias ou discussões que se deseja promover, indexar, "subir" nas redes sociais, inserindo o símbolo da cerquilha (\#) antes da palavra, frase ou expressão (Pinto et al., 2020). Quando a combinação é publicada, transforma-se em um hiperlink que leva para uma página com outras publicações relacionadas ao mesmo tema, promovendo interação de pessoas interessadas naquele assunto.

A escolha pelahashtag \#zerocovid19 resultou da pesquisa nas plataformas Instagram, Twitter, Youtube e Pinterest, realizada entre os dias 14 e 16 de abril de 2020. No momento da investigação, a hashtag apresentava-se altamente divulgada e repostada pelos usuários da rede, além de ser de fácil compreensão para o público em geral. Todo o material divulgado nas redes sociais, adotadas pelo projeto, também reproduziu a hashtag, de forma a colaborar com o processo de visibilidade dos 
Research, Society and Development, v. 11, n. 2, e33511225702, 2022

(CC BY 4.0) | ISSN 2525-3409 | DOI: http://dx.doi.org/10.33448/rsd-v11i2.25702

vídeos e com a divulgação do projeto no ciberespaço. Mesmo que o acesso não seja universal, as mídias sociais tem atingido grande massa da população brasileiro, exercendo protagonismo que se destacou com a pandemia (Pinto, 2019)

No intuito de estruturar melhor a divulgação do material produzido, os vídeos foram agrupados em três eixos temáticos sendo o primeiro eixo intitulado "Vídeos para os Agentes Comunitários de Saúde", o segundo "Autocuidado do profissional" e o último "Triagem para casos suspeitos "A divulgação foi realizada concomitantemente para os serviços de APS municipais, academia e redes sociais. O trabalho de educação permanente em saúde na Estratégia de Saúde da Família, em especial com os ACS, tem avançado deste a implantação deste modelo de atenção, mas ainda apresenta desafios como a distância entre a gestão do cuidado e desta educação, o modos de trabalho ainda produtivista presente na APS sem avaliação do trabalho e problematização da realidade para mudanças no processo de trabalho (Santos et al., 2021).

$\mathrm{Na}$ academia, a disseminação da inovação tecnológica foi realizada pela Pró-reitoria de Extensão e Assuntos Comunitários (PROEX) e a Assessoria de Comunicação Social da Universidade Federal de São João del Rei (ASCOM/UFSJ). A divulgação do material foi feita por meio do site e demais veículos oficiais de informação da Instituição e um boletim informativo, publicado em junho de 2020.

Visando que o conteúdo do material tivesse um alcance mais amplo, foi proposto o compartilhamento destes vídeos e informações em perfis das redes sociais da Universidade, através de sites e plataformas de mídias sociais como o Instagram e o Facebook, além do compartilhamento de links através do aplicativo de mensagens, o WhatsApp e Messenger. Com as divulgações nas redes sociais ampliou-se a disseminação dos vídeos e foi possível atingir número maior de usuários, acessos e compartilhamentos das publicações nas redes sociais (Fiocruz, 2020).

Para os serviços, os vídeos foram disponibilizados para a coordenação da APS de cada município acompanhado de um documento informativo sobre os conteúdos abordados em cada vídeo. Foi ressaltado que os vídeos seguiram os passos do Manual do Ministério da Saúde e foram desenvolvidos utilizando uma linguagem fácil, lúdica e confiável para que o ACS consiga acessar antes e durante as visitas domiciliares e também em condições à distância que se fizer necessário (Brasil, 2020).

Alguns municípios divulgaram os vídeos em seus canais de comunicação institucional, principalmente por meio das plataformas de redes sociais, para maior visibilidade e conscientização da população.

Dentre as limitações para este tipo de trabalho destaca-se a heterogeneidade de formação e características de trabalho de cada município que implica na forma como poderia absorver e repassar as informações propostas em vídeo. Sabe-se que em qualquer tipo de capacitação ou educação em serviço a resposta do participante depende de vários fatores como: interesse, capacidade individual de entendimento e absorção, disponibilidade, envolvimento de seus coordenadores entre outros pontos. Com todo o avanço da tecnologia e das mídias sociais, mas com a dimensão do país e suas discrepâncias socioeconômicas, também constatadas nos serviços de saúde quando se fala de Sistema Único de Saúde, este tipo de trabalho em mãos aos ACS, possibilita amenizar e homogeneizar o distanciamento do acesso da população às orientações sobre o cuidado à saúde (Souza et al., 2021).

Frente às necessidades impostas pela pandemia, o material desenvolvido e suas formas de divulgação, apresentaram contribuições para a prática considerando: continuação do trabalho do ACS enquanto sujeito que está presente nas casas de sua área adscrita de responsabilidade e envolvimento e atração do ACS por meio de um material lúdico e de fácil entendimento. Apesar do material estar direcionado ao ACS, seu conteúdo de fácil assimilação pode ser utilizado pela população em geral. Além das redes sociais de todos os envolvidos na pesquisa e da própria UFSJ, o material encontra-se disponível https://ufsj.edu.br/covid19/videos_para_orientar_agentes_de_saude.php (UFSJ, 2020). 


\section{Considerações Finais}

O trabalho possibilitou a realização de parcerias com secretarias municipais de saúde, as quais instrumentalizaram seus ACS com novas formas e tecnologias interativas no enfrentamento à pandemia da COVID-19. Os canais institucionais de comunicação da universidade, bem como as plataformas de mídias sociais, também se tornaram espaço para divulgação. A pandemia catalisou novas formas de se fazer educação permanente para os profissionais de saúde, em especial ao ACS, assim como educação em saúde para a população em geral.

\section{Agradecimentos}

À Pró-Reitoria de Extensão e Assuntos Comunitários da Universidade Federal de São João del-Rei.

\section{Referências}

Aquino, E. M. L., Silveira, I. H., Pescarini, J. M., Aquino, R., \& de Souza-Filho, J. A. (2020). Social distancing measures to control the COVID-19 pandemic: Potential impacts and challenges in Brazil. Ciencia e Saude Coletiva, 25, 2423-2446.

Bavel, J.J.V., Baicker, K., Boggio, P.S., Capraro, V., Cichocka, A., Cikara, M., Crockett, M. J., Crum, A. J., Douglas, K. M., Druckman, J. N., Drury, J., Dube, O., Ellemers, N., Finkel, E. J., Fowler, J. H., Gelfand, M., Han, S., Haslam, S. A., Jetten, J., Kitayama, S., Mobbs, D., Napper, L. E., Packer, D. J., Pennycook, G., Peters, E., Petty, R. E., Rand, D. G., Reicher, S. D., Schnall, S., Shariff, A., Skitka, L. J., Smith, S. S., Sunstein, C. R., Tabri, N., Tucker, J. A., van der Linden, S., van Lange, P., Weeden, K. A., Wohl, M. J. A., Zaki, J., Zion, S. R., \& Robb Willer. (2020). Using social and behavioural science to support COVID-19 pandemic response. Nature Human Behavior 4, 460-471.

Brasil. Ministério da Saúde. (2020). Recomendações para adequação das ações dos agentes comunitários de saúde frente à atual situação epidemiológica referente à COVID-19. Secretaria de Atenção Primária à Saúde.

Carraro, M. R. S., Ostemberg, E., \& dos Santos, P. K. (2020). As tecnologias digitais na educação e nos processos educativos durante a pandemia do COVID19. Educação Por Escrito, 11(2), e38859-e38859.

Costa, C. S., \& Mattos, F. R. P. (2016). Tecnologia na sala de aula em relatos de professores. Editora CRV.

Dalmolin, A., Girardon-Perlini, N. M. O., Coppetti, L. de C., Rossato, G. C., Gomes, J. S., \& Silva, M. E. N. da. (2016). Vídeo educativo como recurso para educação em saúde a pessoas com colostomia e familiares. Revista Gaúcha de Enfermagem, 37(spe), e68373.

Dutra Pillar A., \& Campos, J. (2021). Narrativas audiovisuais na educação: a videoarte Anima2 e seus sentidos. PÓS: Revista do Programa de Pós-graduação em Artes da EBA/UFMG, 11(21):168-90.

Fiocruz - Instituto Fernandes Figueira (2020). O papel das redes sociais durante a pandemia. Disponível em: http://www.iff.fiocruz.br/index.php/8noticias/675-papel-redes-sociais

Faria, C. C. M. V., \& Paiva, C. H. A. (2020). O trabalho do agente comunitário de saúde e as diferenças sociais no território. Trabalho, educação e saúde, 18(suppl 1):e0025183.

Gonçalves Caldeira Brant Losekann, R., \& Cardoso Mourão, H. (2020). Desafios Do Teletrabalho Na Pandemia Covid-19: Quando O Home Vira Office. Caderno de Administração, 28, 71-75.

Jesus Gonçalves, C. F., Temporim, D. L. S., Santos Mota, I., Oliveira, I. A., Santos, M. de S., \& Souza, R. (2020). Ensino-aprendizagem de línguas utilizando Zoom e Facebook: uma realidade possível. H2D| Revista de Humanidades Digitais, 2(2).

Junior, V. B. S., \& Monteiro, J. C. S. (2020). Educação e COVID-19: as tecnologias digitais mediando a aprendizagem em tempos de pandemia education and COVID-19. Revista Encantar - Educação, Cultura e Sociedade, 2, 1-15.

Machado, A. (2007). Made in Brasil: três décadas do vídeo brasileiro. Editora Iluminuras Ltda.

Maciazeki-Gomes, R. C., Souza, C. D., Baggio, L., \& Wachsssia, F. O trabalho do agente comunitário de saúde na perspectiva da educação popular em saúde: possibilidades e desafios. Ciência \& Saúde Coletiva, 21(5), 1637-1646.

Maciel, F. B. M., Santos, H. L. P. C., Carneiro, R. A. da S., de Souza, E. A., Prado, N. M. de B. L., \& Teixeira, C. F. S. (2020). Agente comunitário de saúde: Reflexões sobre o processo de trabalho em saúde em tempos de pandemia de Covid-19. Ciencia e Saude Coletiva, 25, 4185-95.

Pinto, P. A., Brasileiro, F. S., Antunes, M. J. L. \& Almeida, A. M. P. (2020). COVID-19 no Instagram: práticas de comunicação estratégica das autoridades de saúde durante a pandemia. Comunicação Pública,15(29).

Pinto, P. (2019). Marketing social e digital do Ministério da Saúde no Instagram: estudo de caso sobre aleitamento materno. Revista Eletrônica de Comunicação, Informação e Inovação em Saúde, 13(4). 
Research, Society and Development, v. 11, n. 2, e33511225702, 2022

(CC BY 4.0) | ISSN 2525-3409 | DOI: http://dx.doi.org/10.33448/rsd-v11i2.25702

Rodrigues, S. B., Assis, G. D. P., Silva, B. S., Oliveira, G. C. C. F., Tavares, L. O. de M., Amaral, G. G., Oliveira, V. C., \& Guimarães, E. A. de A. (2021). Simulação realística na capacitação de profissionais de enfermagem em sala de vacinação. Research, Society and Development, $10(3)$, e20810313314.

Santos, A. R., Santos, R. M. M., Franco, T.B., Matumoto S., \& Vilela, A.B.A. (2021). Educação permanente na estratégia saúde da família: potencialidades e ressignificações. Revista de enfermagem UFPE on line, $15: \mathrm{e} 245355 \mathrm{~T}$

Souza, L. P. S. e, Souza, A. G. de, \& Tirelli, C. (2021). COVID-19 no Brasil: seguimos no mesmo mar, mas não nos mesmos barcos. Comunicação Em Ciências Da Saúde, 31(03), 41-8.

Universidade Federal de São João del-Rei - UFSJ. (2020). Criação e desenvolvimento de vídeos educacionais para o trabalho do Agente Comunitário de Saúde (ACS) com a comunidade, no enfrentamento à epidemia de Covid-19. Disponível em: https://ufsj.edu.br/covid19/videos_para_orientar_agentes_de_saude.php 